\title{
Envelope Level Crossing Rate and Average Fade Duration of Nonisotropic Vehicle-to-Vehicle Ricean Fading Channels
}

\author{
Xiang Cheng, Senior Member, IEEE, Cheng-Xiang Wang, Senior Member, IEEE, Bo Ai, Senior Member, IEEE, \\ and Hadi Aggoune, Senior Member, IEEE
}

\begin{abstract}
This paper proposes a generic geometry-based stochastic model for nonisotropic scattering vehicle-to-vehicle (V2V) Ricean fading channels. With the proposed model, the level crossing rate (LCR) and average fade duration (AFD) are derived. The resultant expressions are sufficiently general and subsume many well-known existing LCRs and AFDs as special cases. The derived LCR and AFD are further investigated in terms of some important parameters, e.g., the shape of the scattering region (two-ring or ellipse), mean angle, angle spread, and directions of movement of the Tx and Rx (same or opposite direction). More importantly, in this paper, the impact of the vehicular traffic density on the LCR and AFD for nonisotropic scattering V2V Ricean fading channels is investigated for the first time. Excellent agreement is observed between the theoretical LCRs/AFDs and corresponding measured data, thus demonstrating the validity and utility of the proposed model.
\end{abstract}

Index Terms-Average fade duration (AFD), level crossing rate (LCR), nonisotropic scattering environments, Ricean fading, vehicle-to-vehicle (V2V) channels.

Manuscript received March 14, 2013; revised June 8, 2013; accepted June 28, 2013. Date of publication August 21, 2013; date of current version January 31, 2014. This work was supported in part by the National 973 Project under Grant 2013CB336700; by the National Natural Science Foundation of China under Grant 61101079 and Grant 61222105; by the Science Foundation for the Youth Scholar of the Ministry of Education of China under Grant 20110001120129; by the Open Research Fund of the National Mobile Communications Research Laboratory, Southeast University, under Grant 2012D06; by the Ministry of Transport of China under Grant 2012-364-X03-104; by the Key Laboratory of Cognitive Radio and Information Processing (Guilin University of Electronic Technology) through the Ministry of Education of China under Grant 2013KF01; by the Sensor Networks and Cellular Systems Research Center, University of Tabuk, under the grant from the Ministry of Higher Education of Saudi Arabia; by Beijing Municipal Natural Science Foundation under Grant 4112048; by the Fundamental Research Funds for the Central Universities under Grant 2010JBZ008; and by the Key Project of the Ministry of Railways of China under Grant 2012X008-A. The Associate Editor for this paper was L. Yang. (Corresponding author: C. $-X$. Wang.)

$\mathrm{X}$. Cheng is with the School of Electronics Engineering and Computer Science, Peking University, Beijing 100871, China (e-mail: xiangcheng@pku. edu.cn).

C.-X. Wang is with the School of Information Science and Engineering, Shandong University, Jinan 250100, China, and also with the Joint Research Institute for Signal and Image Processing, School of Engineering and Physical Sciences, Heriot-Watt University, Edinburgh EH14 4AS, U.K. (e-mail: chengxiang.wang@hw.ac.uk).

B. Ai is with the State Key Laboratory of Rail Traffic Control and Safety, Beijing Jiaotong University, Beijing 100044, China (e-mail: boai@bjtu.edu.cn).

H. Aggoune is with the Sensor Networks and Cellular Systems Research Center, University of Tabuk, Tabuk 71491, Saudi Arabia (e-mail: haggoune. sncs@ut.edu.sa).

Color versions of one or more of the figures in this paper are available online at http://ieeexplore.ieee.org.

Digital Object Identifier 10.1109/TITS.2013.2274618

\section{INTRODUCTION}

$\mathbf{I}$ N MOBILE communications with time-variant fading, e.g., vehicular communications in intelligent transportation systems (ITSs) [1], [2], two important second-order statistics associated with envelope fading are the envelope level crossing rate (LCR) and average fade duration (AFD). The LCR describes how often the envelope crosses a certain threshold, whereas AFD indicates how long the envelope stays below a given threshold. The LCR and AFD characterize various aspects of the dynamic temporal behavior of envelope fluctuations. Therefore, they can be used to investigate the disturbing effects of signal fades and consequently appear in a variety of applications.

In recent years, vehicle-to-vehicle $(\mathrm{V} 2 \mathrm{~V})$ communications have received increasing attention and have been considered as an integral part of the next-generation ITSs [4]-[8]. The important benefits of $\mathrm{V} 2 \mathrm{~V}$ communications in ITSs include its potential in improving road safety, enhancing traffic efficiency, and offering infotainment. Such V2V systems consider that both the transmitter (Tx) and receiver ( $\mathrm{Rx})$ are in motion and equipped with low-elevation antennas. To analyze and design a V2V system, it is necessary to have detailed knowledge of the V2V channel and its statistical properties. As mentioned in [9] and [10], in terms of the modeling approach, V2V channel models can be categorized as geometry-based deterministic models, nongeometrical stochastic models, and geometry-based stochastic models (GBSMs) that can be further classified as regular-shaped GBSMs (RS-GBSMs) and irregular-shaped GBSMs. RS-GBSMs assume that all effective scatterers are located on a regular shape [e.g., one/two-ring (TR) or ellipse (EL)] and can often preserve the mathematical tractability. Akki and Haber [11] first proposed an RS-GBSM for narrow-band 2-D isotropic scattering single-input singleoutput (SISO) V2V Rayleigh fading channels. TR-based RSGBSMs were studied in [12] and [13] for narrow-band 2-D nonisotropic multiple-input multiple-output (MIMO) V2V channels. The 2-D TR narrow-band model in [12] was further extended to a 3-D two-cylinder narrow-band and two-concentric-cylinder wideband model in [14] and [15], respectively. Recently, new generic nonisotropic MIMO V2V RSGBSMs have been also proposed for narrow-band [16] and wideband channels [17].

However, all the aforementioned RS-GBSMs focused on the investigation of correlation properties and power 
spectral densities. Although the LCR and AFD are of extreme importance for a V2V channel due to its greater dynamics, only a few papers [18]-[21] investigated the LCR and AFD for V2V channels. In [18], the LCR and AFD for isotropic scattering V2V Rayleigh fading channels in 2-D large spatial scale (LSS) scenarios (i.e., the Tx-Rx distance is larger than $1 \mathrm{~km})$ were studied. In [19] and [20], the authors investigated the LCR and AFD for nonisotropic scattering V2V Ricean fading channels in 3-D LSS and moderate spatial scale (MSS) scenarios (i.e., the Tx-Rx distance ranges from $300 \mathrm{~m}$ to $1 \mathrm{~km}$ ) and also gave the corresponding experimental validation. However, none of the two has studied the LCR and AFD in small spatial scale (SSS) scenarios (i.e., the Tx-Rx distance is smaller than $300 \mathrm{~m}$ ), which are currently receiving more and more attention due to the increasing applications of dedicated shortrange communications to V2V communications. Moreover, due to the unique feature of $\mathrm{V} 2 \mathrm{~V}$ communication environments, the impact of the vehicular traffic density (VTD) on channel characteristics in MSS and SSS scenarios cannot be neglected [10], unlike in LSS scenarios. More recently, in [21], we have performed some preliminary work on the impact of VTD on LCR and AFD. However, the work in [21] simply assumed that the moving scatterers and static scatterers have the same Doppler frequency. This unrealistic assumption underestimates the temporal behavior of envelope fluctuations, and thus, the derived LCR and AFD in [21] are inaccurate.

To fill the aforementioned gaps, this paper proposes a new RS-GBSM and derives the corresponding LCR and AFD. The main contributions and novelties of this paper are as follows.

1) We propose a new V2V RS-GBSM that corrects the unrealistic assumption used in [21] by properly capturing different Doppler frequencies for moving scatterers and static scatterers. The newly developed V2V RS-GBSM is suitable for a wide variety of scenarios, i.e., LSS, MSS, and SSS scenarios, thus enabling the analysis of the VTD impact on channel characteristics. This model employs a combined TR model and EL model, where the received signal is constructed as a sum of the line-ofsight (LoS), single-, and double-bounced rays at different energy levels.

2) Based on the developed RS-GBSM, we derive the LCR and AFD for nonisotropic scattering V2V Ricean fading channels. Our analysis shows several limitations in the derivation and investigation of the LCR and AFD in [19], revealing some easily neglected but important issues. We stress that the derived LCR and AFD subsume several existing LCRs and AFDs as special cases, e.g., those in [18] and [22]-[25].

3) Based on the derived LCR and AFD, we study in more detail the behavior of the LCR and AFD in terms of some important parameters and, for the first time, practically investigate the impact of VTD on LCR and AFD. In addition, comparisons of the obtained theoretical LCR and AFD with measurement data in [26] are presented. Excellent agreement between these corroborates the validity and utility of the proposed model.

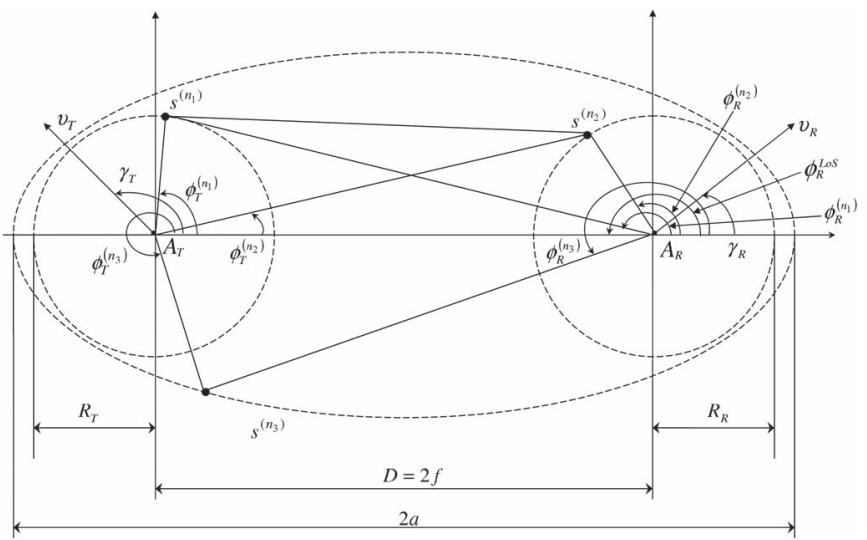

Fig. 1. Generic channel model combining a TR model and an EL model with LoS components and single- and double-bounced rays for a SISO V2V channel.

The remainder of this paper is organized as follows. Section II presents a new generic RS-GBSM for 2-D nonisotropic scattering narrow-band SISO V2V Ricean fading channels. In Section III, based on the proposed model, we derive the corresponding LCR and AFD. Numerical results and analysis, as well as the comparison of the analytical LCR and AFD with the measurement data, are presented in Section IV. Finally, conclusions are drawn in Section V.

\section{NEW GENERIC RS-GBSM FOR NONISOTROPIC V2V RICEAN FADING CHANNELS}

Let us now consider a narrow-band single-user SISO V2V communication system. Both the Tx and Rx are equipped with low-elevation antennas. The propagation scenario is characterized by nonisotropic scattering with a LoS component between the Tx and Rx. Fig. 1 illustrates the geometry of the SISO V2V model, which is the combination of a LoS component, a single-bounce (SB) and double-bounce (DB) TR model, and an SB EL model. The TR model defines two rings of effective scatterers, i.e., one around the Tx and the other around the Rx. Note that effective scatterers are used to represent the effect of many scatterers with similar spatial location. Suppose there are $N_{1}$ effective scatterers around the Tx lying on a ring of radius $R_{T}$ and the $n_{1}$ th $\left(n_{1}=1, \ldots, N_{1}\right)$ effective scatterer is denoted by $s^{\left(n_{1}\right)}$. Similarly, assume there are $N_{2}$ effective scatterers around the Rx lying on a ring of radius $R_{R}$ and the $n_{2}$ th $\left(n_{2}=1, \ldots, N_{2}\right)$ effective scatterer is denoted by $s^{\left(n_{2}\right)}$. For the EL model, $N_{3}$ effective scatterers lie on an EL with the Tx and Rx located at the foci. The semimajor axis of the EL and the $n_{3}$ th $\left(n_{3}=1, \ldots, N_{3}\right)$ effective scatterer are denoted by $a$ and $s^{\left(n_{3}\right)}$, respectively. The distance between the Tx and Rx is $D=2 f$ with $f$ denoting the half length of the distance between the two focal points of the EL. To make the proposed model able to investigate the impact of the VTD on channel statistics, the TR model is used to present moving scatterers, e.g., moving cars around the Tx and Rx, and the EL model is applied to present static scatterers, e.g., static roadside environment. Unlike the previous RS-GBSM in [21], the proposed model actually considers the movement of the moving scatterers by defining different effective velocities of the Tx/Rx with respect to moving scatterers and static 
scatterers. Therefore, the effective velocities of the Tx and Rx with respect to static scatterers are denoted as $v_{T}$ and $v_{R}$ in directions determined by the directions of motion $\gamma_{T}$ and $\gamma_{R}$, respectively. Whereas, the effective velocities of the Tx and Rx with respect to moving scatterers are denoted as $v_{T}^{\prime}$ and $v_{R}^{\prime}$ in directions determined by the directions of motion $\gamma_{T}^{\prime}$ and $\gamma_{R}^{\prime}$, respectively. For clarity purposes, only the effective velocities of the Tx and Rx with regard to static scatterers are shown in Fig. 1. Symbol $\phi_{R_{q}}^{\mathrm{LoS}}$ denotes the angle of arrival (AoA) of a LoS path. The AoA of the wave traveling from the effective scatterer $s^{\left(n_{i}\right)}(i \in\{1,2,3\})$ toward the Rx is denoted by $\phi_{R}^{\left(n_{i}\right)}$. The angle of departure (AoD) of the wave that impinges on the effective scatterer $s^{\left(n_{i}\right)}$ is designated by $\phi_{T}^{\left(n_{i}\right)}$.

From the above model, the received complex fading envelope is the superposition of the LoS component and diffuse components, consisting of single- and double-bounced rays, and can be expressed as

$$
\begin{aligned}
h(t) & =h^{\mathrm{LoS}}(t)+h^{\mathrm{DIF}}(t) \\
& =h^{\mathrm{LoS}}(t)+h^{\mathrm{SB}}(t)+h^{\mathrm{DB}}(t)
\end{aligned}
$$

where $h^{\mathrm{LoS}}(t), h^{\mathrm{SB}}(t)$, and $h^{\mathrm{DB}}(t)$ are expressed in (2a)-(2b), shown at the bottom of the page. $I=3 ; f_{T_{\max }}=v_{T} / \lambda$ and $f_{R_{\max }}=v_{R} / \lambda$ are the maximum Doppler frequencies of the Tx and Rx, respectively, with respect to static scatterers; $f_{T_{\max }}^{\prime}=$ $v_{T}^{\prime} / \lambda$ and $f_{R_{\max }}^{\prime}=v_{R}^{\prime} / \lambda$ are the maximum Doppler frequencies of the Tx and Rx, respectively, with respect to moving scatterers; $f_{T_{\max }}^{1}=f_{T_{\max }}^{2}=f_{T_{\max }}^{\prime} ; f_{T_{\max }}^{3}=f_{T_{\max }} ; f_{R_{\max }}^{1}=$ $f_{R_{\max }}^{2}=f_{R_{\max }}^{\prime}$; and $f_{R_{\max }}^{3}=f_{R_{\max }}$. Symbol $K$ designates the Ricean factor, and $\Omega$ denotes the total power. Parameters $\eta_{\mathrm{SB}_{\mathrm{i}}}$ and $\eta_{\mathrm{DB}}$ specify how much the single- and double-bounced rays contribute to the total scattered power $\Omega /(K+1)$, respectively. Note that these energy-related parameters satisfy $\sum_{i=1}^{I} \eta_{\mathrm{SB}_{i}}+\eta_{\mathrm{DB}}=1$. Phases $\psi_{n_{i}}$ and $\psi_{n_{i}, n_{j}}$ are independent and identically distributed random variables with uniform distributions over $[-\pi, \pi)$, and $\lambda$ is the carrier wavelength. Note that $\operatorname{AoD} \phi_{T}^{\left(n_{i}\right)}$ and AoA $\phi_{R}^{\left(n_{i}\right)}$ are independent random vari- ables for double-bounced rays, whereas they are interdependent for single-bounced rays.

By using the results in [16], we can express the general relationships between the AoD and AoA for the SB TR model as $\phi_{R}^{\left(n_{1}\right)}=\arcsin \left[R_{T} \sin \phi_{T}^{\left(n_{1}\right)} /\left(R_{T}^{2}+D^{2}-2 R_{T} D \cos \phi_{T}^{\left(n_{1}\right)}\right)^{1 / 2}\right]$ and $\phi_{T}^{\left(n_{2}\right)}=\arcsin \left[R_{R} \sin \phi_{R}^{\left(n_{2}\right)} /\left(R_{R}^{2}+D^{2}+2 R_{R} D \cos \phi_{R}^{\left(n_{2}\right)}\right)^{1 / 2}\right]$ and for the EL model as $\phi_{T}^{\left(n_{3}\right)}=\arcsin \left[b^{2} \sin \phi_{R}^{\left(n_{3}\right)} /\left(a^{2}+\right.\right.$ $\left.\left.f^{2}+2 a f \cos \phi_{R}^{\left(n_{3}\right)}\right)\right]$, where $b$ denotes the semiminor axis of the EL and equality qq holds. The above exact relationships are sufficiently general and thus suitable for a wide variety of scenarios. For LSS and MSS scenarios, since assumption $D \gg$ $\max \left\{R_{T}, R_{R}\right\}$ holds, the aforementioned exact relationships of $\phi_{R}^{\left(n_{1}\right)} \leftrightarrow \phi_{T}^{\left(n_{1}\right)}$ and $\phi_{R}^{\left(n_{2}\right)} \leftrightarrow \phi_{T}^{\left(n_{2}\right)}$ can reduce to the following widely used approximate relationships [19]: $\phi_{R}^{\left(n_{1}\right)} \approx \pi-$ $\Delta_{T} \sin \phi_{T}^{\left(n_{1}\right)}$ and $\phi_{T}^{\left(n_{2}\right)} \approx \Delta_{R} \sin \phi_{R}^{\left(n_{2}\right)}$, respectively, with $\Delta_{T} \approx R_{T} / D$ and $\Delta_{R} \approx R_{R} / D$.

Since we assume that the numbers of effective scatterers $N_{i}$ tend to be infinite, the discrete $\operatorname{AoA} \phi_{R}^{\left(n_{i}\right)}$ and $\operatorname{AoD} \phi_{T}^{\left(n_{i}\right)}$ can be replaced by continuous random variables $\phi_{R}^{\mathrm{SB}_{\mathrm{i}}}$ and $\phi_{T}^{\mathrm{SB}_{\mathrm{i}}}$, respectively. In this paper, the von Mises probability density function (PDF) [27] is used to describe $\mathrm{AoA} / \mathrm{AoD}$. The von Mises PDF is defined as $f(\phi) \triangleq \exp [k \cos (\phi-\mu)] /\left[2 \pi I_{0}(k)\right]$, where $\phi \in[-\pi, \pi), I_{0}(\cdot)$ is the zeroth-order modified Bessel function of the first kind, $\mu \in[-\pi, \pi)$ accounts for the mean value of the angle $\phi$, and $k(k \geq 0)$ is a real-valued parameter that controls the angle spread of the angle $\phi$. For $k=0$ (isotropic scattering), the von Mises PDF reduces to the uniform distribution, whereas for $k>0$ (nonisotropic scattering), the von Mises PDF approximates different distributions based on the values of $k$ [27]. In this paper, for the angles of interest, e.g., the $\mathrm{AoD} \phi_{T}^{\mathrm{SB}}$ for the one ring around the Tx, the AoA $\phi_{R}^{\mathrm{SB}_{2}}$ for the one ring around the Rx, and the AoA $\phi_{R}^{\mathrm{SB}_{3}}$ for the EL, we will replace the relevant parameters $\mu$ and $k$ of the von Mises PDF in (6) as $\mu_{T}^{\mathrm{TR}}$ and $k_{T}^{\mathrm{TR}}, \mu_{R}^{\mathrm{TR}}$ and $k_{R}^{\mathrm{TR}}$, and $\mu_{R}^{\mathrm{EL}}$ and $k_{R}^{\mathrm{EL}}$, respectively.

Based on the same approach on how to adjust the important model parameters (i.e., distance $D$, power-related parameters

$$
\begin{aligned}
h^{\mathrm{LoS}}(t) & =\sqrt{\frac{K \Omega}{K+1}} e^{j 2 \pi f_{T_{\max }} t \cos \left(\pi-\phi_{R}^{\mathrm{LOS}}+\gamma_{T}\right)} e^{2 \pi f_{R_{\max }} t \cos \left(\phi_{R}^{\mathrm{LoS}}-\gamma_{R}\right)} \\
h^{\mathrm{SB}}(t) & =\sum_{i=1}^{I} h^{\mathrm{SB}_{\mathrm{i}}}(t) \\
& =\sum_{i=1}^{I} \sqrt{\frac{\eta_{\mathrm{SB}_{i}} \Omega}{K+1}} \lim _{N_{i} \rightarrow \infty} \sum_{n_{i}=1}^{N_{i}} \frac{1}{\sqrt{N_{i}}} e^{j \psi_{n_{i}}} e^{j\left[2 \pi f_{T_{\max }}^{i} t \cos \left(\phi_{T}^{\left(n_{i}\right)}-\gamma_{T}^{i}\right)+2 \pi f_{R_{\max }}^{i} t \cos \left(\phi_{R}^{\left(n_{i}\right)}-\gamma_{R}^{i}\right)\right]} \\
h^{\mathrm{DB}}(t) & =\sqrt{\frac{\eta_{\mathrm{DB}} \Omega}{K+1}} \lim _{N_{1}, N_{2} \rightarrow \infty} \sum_{n_{1}, n_{2}=1}^{N_{1}, N_{2}} \frac{1}{\sqrt{N_{1} N_{2}}} e^{j \psi_{n_{1}, n_{2}}} e^{j\left[2 \pi f_{T_{\max }}^{\prime} t \cos \left(\phi_{T}^{\left(n_{1}\right)}-\gamma_{T}^{\prime}\right)+2 \pi f_{R_{\max }}^{\prime} t \cos \left(\phi_{R}^{\left(n_{2}\right)}-\gamma_{R}^{\prime}\right)\right]}
\end{aligned}
$$


$\eta_{\mathrm{SB}_{\mathrm{i}}}$ and $\eta_{\mathrm{DB}}$, and Ricean factor $K$ ) as in [16], the proposed model is suitable for a wide variety of scenarios, i.e., LSS $(D \geq 1000 \mathrm{~m})$, MSS $(300<D<1000 \mathrm{~m})$, and SSS scenarios $(D \leq 300 \mathrm{~m})$. It is worth mentioning that the assumption $D \gg$ $\max \left\{R_{T}, R_{R}\right\}$ widely used in LSS and MSS scenarios cannot be applied to SSS scenarios since distance $D$ is relatively small. As mentioned in [16], for a low VTD environment, the value of $K$ is large and the power-related parameters fulfill $\eta_{\mathrm{SB}_{3}}>\max \left\{\eta_{\mathrm{SB}_{1}}, \eta_{\mathrm{SB}_{2}}\right\}>\eta_{\mathrm{DB}}$, whereas for a high VTD environment, we have a relatively small $K$ and $\eta_{\text {DB }}>$ $\max \left\{\eta_{\mathrm{SB}_{1}}, \eta_{\mathrm{SB}_{2}}, \eta_{\mathrm{SB}_{3}}\right\}$.

\section{ENVELOPE LCR AND AFD FOR V2V CHANNELS}

In this section, based on the proposed RS-GBSM in (1) and (2a)-(2c), we will derive the LCR and AFD for a nonisotropic scattering environment. LCR $L_{\xi}\left(r_{l}\right)$ is by definition the average number of crossings per second that the signal envelope $\xi(t)=|h(t)|$ crosses a specified level $r_{l}$ with positive/negative slope. Using the traditional PDF-based method [28], we derive the general expression of the LCR for V2V Ricean fading channels as

$$
\begin{aligned}
& L_{\xi}\left(r_{l}\right) \\
& =\frac{2 r_{l}}{\pi^{3 / 2}} \sqrt{\frac{B(K+1)}{b_{0}}} e^{-K-(K+1) r_{l}^{2}} \\
& \quad \times \int_{0}^{\pi / 2} \cosh \left(2 \sqrt{K(K+1)} r_{l} \cos \theta\right) \\
& \quad \times\left[e^{-\left(\frac{\varsigma \zeta \sin \theta}{\sqrt{2 B}}\right)^{2}}+\sqrt{\frac{\pi}{2 B}} \varsigma \zeta \sin \theta \cdot \operatorname{erf}\left(\frac{\varsigma \zeta \sin \theta}{\sqrt{2 B}}\right)\right] d \theta
\end{aligned}
$$

where $\cosh (\cdot)$ is the hyperbolic cosine, $\operatorname{erf}(\cdot)$ denotes the error function, $B=b_{2}-b_{1}^{2} / b_{0}, \quad \zeta=\sqrt{K \Omega /(K+1)}$, and $\varsigma=E+b_{1} / b_{0}$ with $E=2 \pi f_{T_{\max }} \cos \left(\pi-\phi_{R}^{\mathrm{LOS}}+\gamma_{T}\right)+$ $2 \pi f_{R_{\max }} \cos \left(\phi_{R}^{\mathrm{LoS}}-\gamma_{R}\right)$. Finally, the key parameters $b_{m}$ $(m=0,1,2)$ are defined as $b_{m}=\left.\left(d^{m} \rho_{h^{\mathrm{DIF}}}(\tau) / 2 j^{m} d \tau^{m}\right)\right|_{\tau=0}$ with $\rho_{h^{\mathrm{DIF}}}(\tau)=\mathbf{E}\left[h^{\mathrm{DIF}}(t) h^{\mathrm{DIF} *}(t-\tau)\right] / \Omega$, where $j^{2}=-1$, $\rho_{h \mathrm{DIF}}(\tau)$ is the time autocorrelation function (ACF) of the diffuse component $h^{\mathrm{DIF}}(t)$ of the complex channel fading envelope, $(\cdot)^{*}$ denotes the complex conjugate operation, and $\mathbf{E}[\cdot]$ designates the statistical expectation operator.

Before we derive the key parameters $b_{m}(m=0,1,2)$ of the LCR in (3), let us first clarify the relationship of different LCR expressions for Ricean fading channels, i.e., (3) in this paper, [19, eq. (6)] or [20, eq. (43)], and [22, eq. (2.99)]. These expressions were given separately in different papers and should be used carefully for different propagation environments. It is obvious that (3) is a general expression and can reduce to [19, eq. (6)] and [20, eq. (43)] by setting $E=0$. If we further assume that $b_{1}=0$ in (3) (it follows that $\varsigma=0$ ), we can obtain the closed-form LCR expression [22, eq. (2.99)]. We can therefore conclude that, for a nonisotropic scattering environment $\left(b_{1} \neq 0\right)$ with a time-dependent LoS component $(E \neq 0)$, we can only choose (3) as the LCR expression. Whereas, for a nonisotropic scattering environment with a time-independent LoS component $(E=0)$, either (3) here with $E=0$ or [19, eq. (6)] and [20, eq. (43)] can be used. However, in [19] and [20], the authors have misused (6) and (43), respectively, as the expressions to study the LCR for a nonisotropic scattering environment but with a time-dependent LoS component. The LCR expression in [22, eq. (2.99)] is suitable only for an isotropic scattering environment $\left(b_{1}=0\right)$ with a time-independent LoS component.

\section{A. Derivation of $b_{m}$ for a Wide Variety of Scenarios}

Based on the presented model in Section II, we now derive the key parameters $b_{m}$. Substituting (1), (2b), and (2c) into the expression of $b_{m}$ and setting $m=0$, we can obtain parameter $b_{0}$ as

$$
b_{0}=b_{0}^{\mathrm{SB}_{1}}+b_{0}^{\mathrm{SB}_{2}}+b_{0}^{\mathrm{SB}_{3}}+b_{0}^{\mathrm{DB}}=\frac{1}{2(K+1)}
$$

where $b_{0}^{\mathrm{SB}_{1}}=\eta_{\mathrm{SB}_{1}} /(2(K+1)), \quad b_{0}^{\mathrm{SB}_{2}}=\eta_{\mathrm{SB}_{2}} /(2(K+1))$, $b_{0}^{\mathrm{SB}_{3}}=\eta_{\mathrm{SB}_{3}} /(2(K+1))$, and $b_{0}^{\mathrm{DB}}=\eta_{\mathrm{DB}} /(2(K+1))$. Note that (4) corrects the expression of $b_{0}$ in [19, eq. (10)], where $b_{0}=(1 / K+1)$. Similarly, we can express parameters $b_{1}$ and $b_{2}$ as

$$
b_{m}=b_{m}^{\mathrm{SB}_{1}}+b_{m}^{\mathrm{SB}_{2}}+b_{m}^{\mathrm{SB}_{3}}+b_{m}^{\mathrm{DB}}, \quad m=1,2 .
$$

Considering the von Mises PDFs for the TR model, we can express parameters $b_{m}^{\mathrm{SB}_{1(2)}}(m=1,2)$ as

$$
\begin{aligned}
& b_{1}^{\mathrm{SB}_{1(2)}}= b_{0}^{\mathrm{SB}_{1(2)}} \int_{-\pi}^{\pi} \frac{e^{k_{T(R)}^{\mathrm{TR}} \cos \left(\phi_{T(R)}^{\mathrm{SB}_{1(2)}}-\mu_{T(R)}^{\mathrm{TR}}\right)}}{I_{0}\left(k_{T(R)}^{\mathrm{TR}}\right)} \\
& \times\left[f_{T_{\max }} \cos \left(\phi_{T}^{\mathrm{SB}_{1(2)}}-\gamma_{T}\right)\right. \\
&\left.+f_{R_{\max }} \cos \left(\phi_{R}^{\mathrm{SB}_{1(2)}}-\gamma_{R}\right)\right] d \phi_{T(R)}^{\mathrm{SB}_{1(2)}} \\
& b_{2}^{\mathrm{SB}_{1(2)}}= b_{0}^{\mathrm{SB}_{1(2)}} \int_{-\pi}^{\pi} \frac{2 \pi e^{k_{T(R)}^{\mathrm{TR}} \cos \left(\phi_{T(R)}^{\mathrm{SB}_{1(2)}}-\mu_{T(R)}^{\mathrm{TR}}\right)}}{I_{0}\left(k_{T(R)}^{\mathrm{TR}}\right)} \\
& \times\left[f_{T_{\max }} \cos \left(\phi_{T}^{\mathrm{SB}_{1(2)}}-\gamma_{T}\right)\right. \\
&\left.\quad+f_{R_{\max }} \cos \left(\phi_{R}^{\mathrm{SB}_{1(2)}}-\gamma_{R}\right)\right]^{2} d \phi_{T(R)}^{\mathrm{SB}_{1(2)}} .
\end{aligned}
$$

Based on the general relationship between the AoA and AoD for the SB TR model, the parameters $\phi_{R}^{\mathrm{SB}_{1}}$ and $\phi_{T}^{\mathrm{SB}_{2}}$ in (6a) and (6b) can be expressed by $\phi_{T}^{\mathrm{SB}_{1}}$ and $\phi_{R}^{\mathrm{SB}_{2}}$, respectively. Similarly, considering the von Mises PDF for the EL model, we can express parameters $b_{m}^{\mathrm{SB}_{3}}(m=1,2)$ as

$$
\begin{aligned}
b_{1}^{\mathrm{SB}_{3}}= & b_{0}^{\mathrm{SB}_{3}} \int_{-\pi}^{\pi} \frac{e^{k_{R}^{\mathrm{ER}} \cos \left(\phi_{R}^{\mathrm{SB}_{3}}-\mu_{R}^{\mathrm{ER}}\right)}}{I_{0}\left(k_{R}^{\mathrm{ER}}\right)} \\
& \times\left[f_{T_{\max }} \cos \left(\phi_{T}^{\mathrm{SB}_{3}}-\gamma_{T}\right)\right.
\end{aligned}
$$




$$
\begin{aligned}
& \left.+f_{R_{\max }} \cos \left(\phi_{R}^{\mathrm{SB}_{3}}-\gamma_{R}\right)\right] d \phi_{R}^{\mathrm{SB}_{3}} \\
& b_{2}^{\mathrm{SB}_{3}}=b_{0}^{\mathrm{SB}_{3}} \int_{-\pi}^{\pi} \frac{2 \pi e^{k_{R}^{\mathrm{ER}}} \cos \left(\phi_{R}^{\mathrm{SB}_{3}}-\mu_{R}^{\mathrm{ER}}\right)}{I_{0}\left(k_{R}^{\mathrm{ER}}\right)} \\
& \times\left[f_{T_{\max }} \cos \left(\phi_{T}^{\mathrm{SB}_{3}}-\gamma_{T}\right)\right. \\
& \left.+f_{R_{\max }} \cos \left(\phi_{R}^{\mathrm{SB}_{3}}-\gamma_{R}\right)\right]^{2} d \phi_{R}^{\mathrm{SB}_{3}} .
\end{aligned}
$$

According to the general relationship between the AoA and AoD for the EL model, the parameter $\phi_{T}^{\mathrm{SB}_{3}}$ in (7a) and (7b) can be expressed as a function of $\phi_{R}^{\mathrm{SB}_{3}}$. Considering the von Mises PDF for the TR model and applying the following equalities $\int_{-\pi}^{\pi} e^{a \sin c+b \cos c} d c=2 \pi I_{0}\left(\sqrt{a^{2}+b^{2}}\right)$, $d I_{V}(z) / d z=\left[I_{V-1}(z)+I_{V+1}(z)\right] / 2, \quad$ and $\quad z I_{V-1}(z)-$ $z I_{V+1}(z)=(V+1) I_{V}(z)$ [29], where $I_{V}(\cdot)$ is the $V$ thorder modified Bessel function of the first kind, we can get the following closed-form expressions of parameters $b_{m}^{\mathrm{DB}}(m=1,2)$ :

$$
\begin{aligned}
b_{1}^{\mathrm{DB}}= & b_{0}^{\mathrm{DB}} \frac{2 \pi f_{T_{\max }} \cos \left(\gamma_{T}-\mu_{T}^{\mathrm{TR}}\right) I_{1}\left(k_{T}^{\mathrm{TR}}\right)}{I_{0}\left(k_{T}^{\mathrm{TR}}\right)} \\
& +b_{0}^{\mathrm{DB}} \frac{2 \pi f_{R_{\max }} \cos \left(\gamma_{R}-\mu_{R}^{\mathrm{TR}}\right) I_{1}\left(k_{R}^{\mathrm{TR}}\right)}{I_{0}\left(k_{R}^{\mathrm{TR}}\right)} \\
b_{2}^{\mathrm{DB}}= & b_{0}^{\mathrm{DB}} 4 \pi^{2} f_{T_{\max }^{2}} \frac{1+\cos \left(2\left(\gamma_{T}-\mu_{T}^{\mathrm{TR}}\right)\right) I_{2}\left(k_{T}^{\mathrm{TR}}\right)}{2 I_{0}\left(k_{T}^{\mathrm{TR}}\right)} \\
& +b_{0}^{\mathrm{DB}} 4 \pi^{2} f_{R_{\max }}^{2} \frac{1+\cos \left(2\left(\gamma_{R}-\mu_{R}^{\mathrm{TR}}\right)\right) I_{2}\left(k_{R}^{\mathrm{TR}}\right)}{2 I_{0}\left(k_{R}^{\mathrm{TR}}\right)} \\
& +b_{0}^{\mathrm{DB}} 8 \pi^{2} f_{T_{\max }} f_{R_{\max }} \cos \left(\gamma_{T}-\mu_{T}^{\mathrm{TR}}\right) \\
& \times \cos \left(\gamma_{R}-\mu_{R}^{\mathrm{TR}}\right) \frac{I_{1}\left(k_{T}^{\mathrm{TR}}\right) I_{1}\left(k_{R}^{\mathrm{TR}}\right)}{I_{0}\left(k_{T}^{\mathrm{TR}}\right) I_{0}\left(k_{R}^{\mathrm{TR}}\right)} .
\end{aligned}
$$

Since the derivations of (8a) and (8b) are similar, only a brief outline for the derivation of (8b) is given in Appendix A. Numerical integration methods are needed to evaluate the integrals in (6a)-(7b).

\section{B. Derivation of $b_{m}$ for LSS and MSS Scenarios}

For LSS and MSS scenarios, the widely used assumption $D \gg \max \left\{R_{T}, R_{R}\right\}$ can be used in the derivation of $b_{m}$. This results in the closed-form solution of the integrals in (6a) and (6b), whereas the expressions of other parameters $b_{m}$ remain unchanged. According to the expressions of $\phi_{R}^{\left(n_{1}\right)}$ and $\phi_{T}^{\left(n_{2}\right)}$ and using approximate relations $\sin \chi \approx \chi$ and $\cos \chi \approx 1$, when $\chi$ is small, the parameters $b_{m}^{\mathrm{SB}_{1(2)}}(m=1,2)$ in (6a) and (6b) can be further simplified as

$$
\begin{aligned}
b_{m}^{\mathrm{SB}_{1}}= & b_{0}^{\mathrm{SB}_{1}} \int_{-\pi}^{\pi} \frac{(2 \pi)^{m-1} e^{k_{T}^{\mathrm{TR}} \cos \left(\phi_{T}^{\mathrm{SB}_{1}}-\mu_{T}^{\mathrm{TR}}\right)}}{I_{0}\left(k_{T}^{\mathrm{TR}}\right)} \\
& \times\left[f_{R_{\max }}\left(\Delta_{T} \sin \phi_{T}^{\mathrm{SB}_{1}} \sin \gamma_{R}-\cos \gamma_{R}\right)\right.
\end{aligned}
$$

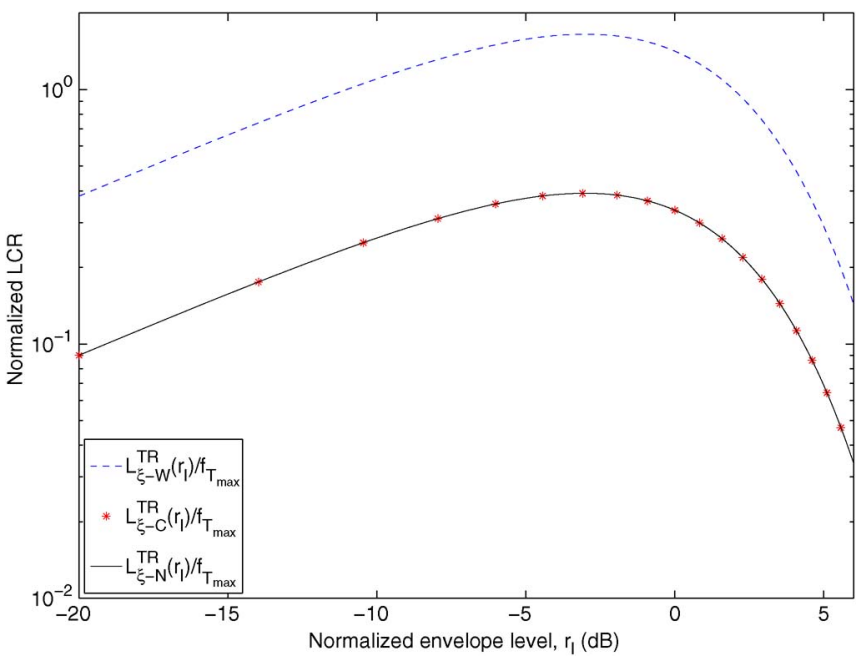

Fig. 2. Comparison of the LCRs $L_{\xi-W}^{\mathrm{TR}}\left(r_{l}\right), L_{\xi-C}^{\mathrm{TR}}\left(r_{l}\right)$, and $L_{\xi-N}^{\mathrm{TR}}\left(r_{l}\right)$.

$$
\begin{gathered}
\left.+f_{T_{\max }} \cos \left(\phi_{T}^{\mathrm{SB}_{1}}-\gamma_{T}\right)\right]^{m} d \phi_{T}^{\mathrm{SB}_{1}} \\
b_{m}^{\mathrm{SB}_{2}}=b_{0}^{\mathrm{SB}_{2}} \int_{-\pi}^{\pi} \frac{(2 \pi)^{m-1} e^{k_{R}^{\mathrm{TR}} \cos \left(\phi_{R}^{\mathrm{SB}}-\mu_{R}^{\mathrm{TR}}\right)}}{I_{0}\left(k_{R}^{\mathrm{TR}}\right)} \\
\times\left[f_{T_{\max }}\left(\Delta_{R} \sin \phi_{R}^{\mathrm{SB}_{2}} \sin \gamma_{T}+\cos \gamma_{T}\right)\right. \\
\left.+f_{R_{\max }} \cos \left(\phi_{R}^{\mathrm{SB}_{2}}-\gamma_{R}\right)\right]^{m} d \phi_{R}^{\mathrm{SB}_{2}} .
\end{gathered}
$$

The integrals in (9a) and (9b) can be further simplified, and thus, the closed-form expressions of parameters $b_{m}^{\mathrm{SB}_{1(2)}}$ can be obtained as (10a) and (10b), shown at the bottom of the next page. In Appendix B, we only provide a brief outline for the derivation of (10b) since the derivations of (10a) and (10b) are similar. Note that the expressions of parameters $b_{m}$ in (6a)-(8b) make the LCR expression in (3) suitable for a wider variety of scenarios, whereas the expressions in (10a) and (10b) limit the application of (3) to only LSS and MSS scenarios. The parameters $b_{m}^{\mathrm{SB}_{1(2)}}$ for 3-D narrow-band and wideband M2M channels were derived in [19] and [20], respectively. The expressions of $b_{m}^{\mathrm{SB}_{1(2)}}$ in [20] can reduce to the ones in [19] by setting $R_{t(r) 1}=R_{t(r) 2}$. If we further assume elevation-related parameters $\beta_{T(R)}=\Delta_{H}=0$ in the closed-form expressions of parameters $b_{n}^{\mathrm{SB}_{1(2)}}$ in [19, eqs. (19) and (21)], the resulting parameters $b_{m}^{\mathrm{SB}_{1(2)}}$ for a 2-D LSS or MSS scenario can be expressed as (11a) and (11b), shown at the next page below (10a) and (10b). It is obvious that the closed-form expressions for the same parameters $b_{m}^{\mathrm{SB}_{1(2)}}$ in (10a)-(11b) are different. To determine which closed-form expressions of $b_{m}^{\mathrm{SB}_{1(2)}}$ are correct, in Fig. 2, we compare the LCRs having the same expression (3) but with different expressions of parameters $b_{m}^{\mathrm{SB}_{1(2)}}$ : 1) $L_{\xi-C}^{\mathrm{TR}}\left(r_{l}\right)$ having $b_{m}^{\mathrm{SB}_{1(2)}}$ calculated by (10a) and (10b); 2) $L_{\xi-W}^{\mathrm{TR}}\left(r_{l}\right)$ having $b_{m}^{\mathrm{SB}_{1(2)}}$ in (11a) and (11b); and 3) $L_{\xi-N}^{\mathrm{TR}}\left(r_{l}\right)$ having the numerically computed $b_{m}^{\mathrm{SB}_{1(2)}}$ in (9a) and (9b). The parameters used in Fig. 2 are $f_{T_{\max }}=f_{R_{\max }}=500 \mathrm{~Hz}, K=0$, $\Delta_{T}=\Delta_{R}=0.01, \mu_{T}^{\mathrm{TR}}=31.2^{\circ}, k_{T}^{\mathrm{TR}}=18.2, \mu_{R}^{\mathrm{TR}}=216.3^{\circ}$, 
and $k_{R}^{\mathrm{TR}}=10.6$. Fig. 2 shows excellent agreement between $L_{\xi-C}^{\mathrm{TR}}\left(r_{l}\right)$ and $L_{\xi-N}^{\mathrm{TR}}\left(r_{l}\right)$, demonstrating the correctness of our derivations (10a) and (10b).

It is worth emphasizing that the LCR $L_{\xi-C}^{\mathrm{TR}}\left(r_{l}\right)$, which is based on the closed-form expressions of parameters $b_{n}^{\mathrm{SB}_{1(2)}}$ in (10a) and (10b), is obtained by applying the widely used assumption $D \gg \max \left\{R_{T}, R_{R}\right\}$ in LSS and MSS scenarios. To fulfill this assumption, the values of parameters $\Delta_{T}$ and $\Delta_{R}$ should be chosen carefully due to the relationships $\Delta_{T} \approx$ $R_{T} / D$ and $\Delta_{R} \approx R_{R} / D$ (in general, the smaller, the better).

$$
\begin{aligned}
& b_{1}^{\mathrm{SB}_{1(2)}}=b_{0}^{\mathrm{SB}_{1(2)}}\left\{2 \pi\left[f_{T(R)_{\max }} \cos \left(\gamma_{T(R)}-\mu_{T(R)}^{\mathrm{TR}}\right)+f_{R(T)_{\max }} \Delta_{T(R)} \sin \mu_{T(R)}^{\mathrm{TR}} \sin \gamma_{R(T)}\right]\right. \\
& \left.\times \frac{I_{1}\left(k_{T(R)}^{\mathrm{TR}}\right)}{I_{0}\left(k_{T(R)}^{\mathrm{TR}}\right)} \mp 2 \pi f_{R(T)_{\max }} \cos \gamma_{R(T)}\right\} \\
& b_{2}^{\mathrm{SB}_{1(2)}}=b_{0}^{\mathrm{SB}_{1(2)}}\left\{2 \pi^{2}\left(2 f_{R(T)_{\max }}^{2} \cos ^{2} \gamma_{R(T)}+f_{T(R)_{\max }}^{2}+f_{R(T)_{\max }}^{2} \Delta_{T(R)}^{2} \sin ^{2} \gamma_{R(T)}+2 f_{T_{\max }} f_{R_{\max }} \Delta_{T(R)} \sin \gamma_{T} \sin \gamma_{R}\right)\right. \\
& \mp 4 \pi^{2}\left[2 f_{T_{\max }} f_{R_{\max }} \cos \gamma_{R(T)} \cos \left(\gamma_{T(R)}-\mu_{T(R)}^{\mathrm{TR}}\right)+f_{R(T)_{\max }}^{2} \Delta_{T(R)} \sin \left(2 \gamma_{R(T)}\right) \sin \mu_{T(R)}^{\mathrm{TR}}\right] \frac{I_{1}\left(k_{T(R)}^{\mathrm{TR}}\right)}{I_{0}\left(k_{T(R)}^{\mathrm{TR}}\right)} \\
& +2 \pi^{2}\left[f_{T(R)_{\max }}^{2} \cos \left(2\left(\gamma_{T(R)}-\mu_{T(R)}^{\mathrm{TR}}\right)\right)-f_{R(T)_{\max }}^{2} \Delta_{T(R)}^{2} \sin ^{2} \gamma_{R(T)} \cos \left(2 \mu_{T(R)}^{\mathrm{TR}}\right)\right. \\
& \left.\left.-2 f_{T_{\max }} f_{R_{\max }} \Delta_{T(R)} \sin \gamma_{R(T)} \sin \left(\gamma_{T(R)}-2 \mu_{T(R)}^{\mathrm{TR}}\right)\right] \frac{I_{2}\left(k_{T(R)}^{\mathrm{TR}}\right)}{I_{0}\left(k_{T(R)}^{\mathrm{TR}}\right)}\right\} .
\end{aligned}
$$

$$
\begin{aligned}
& b_{1}^{\mathrm{SB}_{1(2)}}=b_{0}^{\mathrm{SB}_{1(2)}}\left\{2 \pi\left[f_{T(R)_{\max }} \cos \left(\gamma_{T(R)}-\mu_{T(R)}^{\mathrm{TR}}\right)-f_{R(T)_{\max }} \Delta_{T(R)} \sin \mu_{T(R)}^{\mathrm{TR}} \sin \gamma_{R(T)}\right]\right. \\
& \left.\times \frac{I_{1}\left(k_{T(R)}^{\mathrm{TR}}\right)}{I_{0}\left(k_{T(R)}^{\mathrm{TR}}\right)} \pm 2 \pi f_{R(T)_{\max }} \cos \gamma_{R(T)}\right\} \\
& b_{2}^{\mathrm{SB}_{1(2)}}=b_{0}^{\mathrm{SB}_{1(2)}}\left\{2 \pi^{2}\left(2 f_{R(T) \text { max }}^{2} \cos \gamma_{R(T)}^{2}+\frac{2}{\pi^{2}} f_{T_{\max }} f_{R_{\max }} \Delta_{T(R)} \sin \gamma_{R(T)} \cos \gamma_{T(R)} \mp 4 f_{T_{\max }} \times f_{R_{\max }} \cos \gamma_{R(T)}\right)\right. \\
& +2 \pi^{2}\left(f_{T(R)_{\max }}^{2}+f_{R(T)_{\max }}^{2} \Delta_{T(R)}^{2} \sin \gamma_{R(T)}^{2}\right) \frac{1}{I_{0}\left(k_{T(R)}^{\mathrm{TR}}\right)} \\
& \pm 4 \pi^{2} f_{R(T)_{\max }}^{2} \Delta_{T(R)} \sin \left(2 \gamma_{R(T)}\right) \sin \mu_{T(R)}^{\mathrm{TR}} \frac{I_{1}\left(k_{T(R)}^{\mathrm{TR}}\right)}{I_{0}\left(k_{T(R)}^{\mathrm{TR}}\right)} \\
& -2 \pi^{2}\left[f_{T(R)_{\max }}^{2} \cos \left(2\left(\gamma_{T(R)}-\mu_{T(R)}^{\mathrm{TR}}\right)\right)+f_{R(T)_{\max }}^{2} \Delta_{T(R)}^{2} \sin \gamma_{R(T)}^{2} \cos \left(2 \mu_{T(R)}^{\mathrm{TR}}\right)\right. \\
& \left.\left.+\frac{2}{\pi^{2}} f_{T_{\max }} f_{R_{\max }} \Delta_{T(R)} \sin \gamma_{R(T)} \cos \left(2 \mu_{T(R)}^{\mathrm{TR}}-\gamma_{T(R)}\right)\right] \frac{I_{2}\left(k_{T(R)}^{\mathrm{TR}}\right)}{I_{0}\left(k_{T(R)}^{\mathrm{TR}}\right)}\right\} .
\end{aligned}
$$




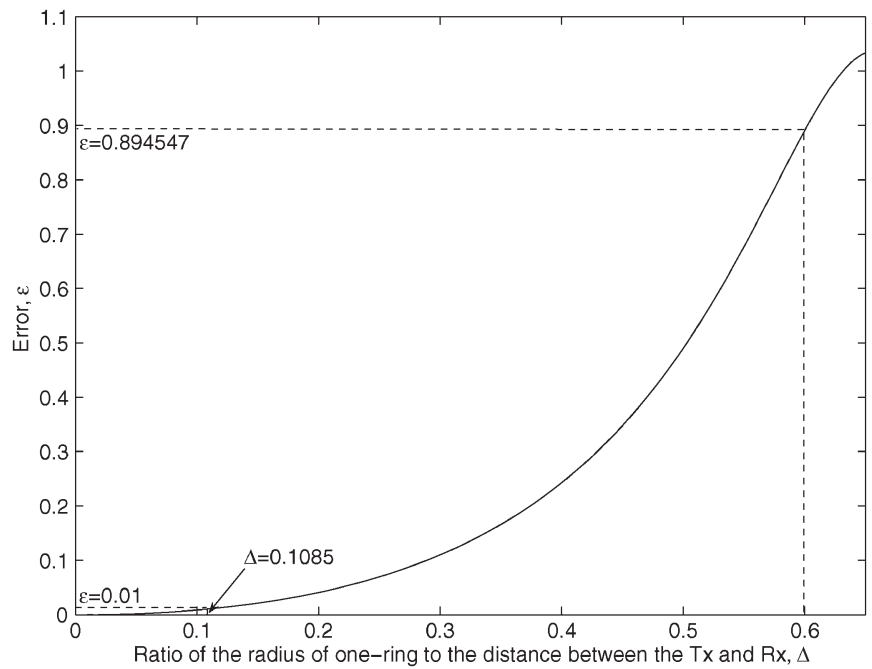

Fig. 3. Error $\varepsilon$ between the LCRs $L_{\xi-G}^{\mathrm{TR}}\left(r_{l}\right)$ and $L_{\xi-C}^{\mathrm{TR}}\left(r_{l}\right)$.

However, the values of parameters $\Delta_{T}$ and $\Delta_{R}$ are chosen comparatively large $\left(\Delta_{T}=\Delta_{R}=0.6\right)$ in [19]. This raises several questions, such as whether $\Delta_{T}=\Delta_{R}=0.6$ violates assumption $D \gg \max \left\{R_{T}, R_{R}\right\}$; if so, how inaccurate the LCR $L_{\xi-C}^{\mathrm{TR}}\left(r_{l}\right)$ is; and whether this inaccuracy is acceptable. To address the above questions, we define an error function to measure the error between the LCR $L_{\xi-G}^{\mathrm{TR}}\left(r_{l}\right)$, having the expression of (3) with the general expressions of parameters $b_{m}^{\mathrm{SB}_{1(2)}}$ in (6a) and (6b), and $L_{\xi-C}^{\mathrm{TR}}\left(r_{l}\right)$ as $\varepsilon=L^{-1} \sum_{l=1}^{L} \mid L_{\xi-G}^{\mathrm{TR}}\left(r_{l}\right)-$ $L_{\xi-C}^{\mathrm{TR}}\left(r_{l}\right)|/| L_{\xi-G}^{\mathrm{TR}}\left(r_{l}\right) \mid$, where $L$ is the total number of the investigated specified level $r_{l}$. Fig. 3 illustrates the error $\varepsilon$ versus the parameter $\Delta\left(\Delta_{T}=\Delta_{R}=\Delta\right)$ by using the same parameters as in Fig. 2. In Fig. 3, the investigated levels $r_{l}$ were obtained by taking $L=200$ equal-distance samples between -20 and $5 \mathrm{~dB}$. As expected, it is shown that the inaccuracy of $L_{\xi-C}^{\mathrm{TR}}\left(r_{l}\right)$ increases with the increase in parameter $\Delta$. From Fig. 3, it is obvious that at $\Delta=0.6$, we have very high error $\varepsilon=$ 0.894547 . This demonstrates that the chosen parameters $\Delta_{T}=$ $\Delta_{R}=0.6$ in [19] may result in extremely inaccurate $L_{\xi-C}^{\mathrm{TR}}\left(r_{l}\right)$. Therefore, it is desirable to propose a criterion on how to choose parameters $\Delta_{T}$ and $\Delta_{R}$ to guarantee the acceptable accuracy of $L_{\xi-C}^{\mathrm{TR}}\left(r_{l}\right)$. In this paper, we assume that the accuracy of $L_{\xi-C}^{\mathrm{TR}}\left(r_{l}\right)$ is acceptable if the error $\varepsilon \leq 0.01$. In such a case, the LCR $L_{\xi-C}^{\mathrm{TR}}\left(r_{l}\right)$ can be applied under the condition that $\Delta_{T}=$ $\Delta_{R} \leq 0.1085$. In other words, when $\Delta_{T}=\Delta_{R}>0.1085$, the LCR $L_{\xi-G}^{\mathrm{TR}}\left(r_{l}\right)$ can only be used instead of $L_{\xi-C}^{\mathrm{TR}}\left(r_{l}\right)$.

AFD $T_{\xi-}\left(r_{l}\right)$ is the average time over which the signal envelope $\xi(t)$ remains below a certain level $r_{l}$. In general, the AFD $T_{\xi-}\left(r_{l}\right)$ for Ricean fading channels is defined by [22]

$$
T_{\xi-}\left(r_{l}\right)=\frac{P_{\xi-}\left(r_{l}\right)}{L_{\xi}\left(r_{l}\right)}=\frac{1-Q\left(\sqrt{2 K}, \sqrt{2(K+1)} r_{l}\right)}{L_{\xi}\left(r_{l}\right)}
$$

where $P_{\xi-}\left(r_{l}\right)$ indicates a cumulative distribution function of $\xi(t)$ with $Q(\cdot, \cdot)$ denoting the generalized Marcum $Q$ function.

Many existing LCRs and AFDs are special cases of the nonisotropic V2V LCR and AFD in (3) and (12), respectively. The simplest case is Clarke's LCR $\sqrt{2 \pi} f_{R_{\max }} r_{l} \exp \left(-r_{l}^{2}\right)$ and
AFD $\left(\exp \left(r_{l}^{2}\right)-1\right) /\left(\sqrt{2 \pi} f_{R_{\max }} r_{l}\right)$ [22], which can be obtained from (3) and (12), respectively, by setting $K=0$ (nonLoS condition), $k_{R}^{\mathrm{TR}}=0$ (isotropic scattering around $\mathrm{Rx}$ ), and $f_{T_{\max }}=k_{T}^{\mathrm{TR}}=\eta_{\mathrm{SB}_{1}}=\eta_{\mathrm{SB}_{3}}=\eta_{\mathrm{DB}}=0$ (fixed Tx, no scattering around Tx). Expressions for other LCRs and AFDs based on the isotropic one-ring model only around the Rx, e.g., those in [23] and [24], can be similarly obtained. The LCR and AFD based on the nonisotropic TR model with single-bounced rays [25] are obtained from (3) and (12), respectively, by setting $K=f_{T_{\max }}=\eta_{\mathrm{SB}_{3}}=\eta_{\mathrm{DB}}=0$. Finally, the LCR and AFD for isotropic V2V Rayleigh channels [18] are obtained from (3) and (12), respectively, by setting $K=k_{T}^{\mathrm{TR}}=k_{R}^{\mathrm{TR}}=\eta_{\mathrm{SB}_{1}}=$ $\eta_{\mathrm{SB}_{2}}=\eta_{\mathrm{SB}_{3}}=0$.

\section{NumeriCAl Results AND ANALYSIS}

In this section, based on the derived LCR and AFD in Section III, these two second-order statistics of the nonisotropic V2V Ricean fading channel are numerically analyzed in more detail in terms of some important parameters, e.g., the scattering assumption (single- or double-bounced rays), shape of the scattering region (TR or EL), mean angle, angle spread, and directions of movement of the Tx and Rx [same direction (SD) or opposite direction (OD)]. In addition, we compare the theoretical results of the LCR and AFD with the measured data in [26]. Without loss of generality, we assume that the effective directions of motion of the $\mathrm{Tx} / \mathrm{Rx}$ with respect to moving scatterers and static scatterers are the same, i.e., $\gamma_{T}=$ $\gamma_{T}^{\prime}$ and $\gamma_{R}=\gamma_{R}^{\prime}$. The following parameters are used for our numerical analysis: $f_{c}=5.2 \mathrm{GHz}, f_{T_{\max }}=f_{R_{\max }}=500 \mathrm{~Hz}$, $f_{T_{\max }}^{\prime}=f_{R_{\max }}^{\prime}=560 \mathrm{~Hz}, a=200 \mathrm{~m}, D=300 \mathrm{~m}$, and $R_{T}=$ $R_{R}=40 \mathrm{~m}$.

Fig. 4 presents the impact of different models (e.g., SB TR model, DB TR model, and SB EL model) and directions of movement of the Tx and Rx (SD or OD) on the LCR and AFD in a nonisotropic scattering scenario, respectively. Results show that the behavior of the LCRs and AFDs for different models is different. We can also observe that the impact of the directions of movement of the Tx and Rx on LCR and AFD exists for single-bounced rays, whereas it disappears for double-bounced rays due to the independent relationship between the AoA and AoD.

Fig. 5 illustrates the LCRs and AFDs of the SB and DB TR models and SB EL model for different nonisotropic scattering scenarios, respectively. It is obvious that the LCR and AFD significantly vary according to the changes of angle spread (related to the values of $k_{T}^{\mathrm{TR}}, k_{R}^{\mathrm{TR}}$, and $k_{R}^{\mathrm{EL}}$ ) and mean angle (related to the values of $\mu_{T}^{\mathrm{TR}}, \mu_{R}^{\mathrm{TR}}$, and $\mu_{R}^{\mathrm{EL}}$ ). Therefore, these two parameters play an important role in the behavior of the LCR and AFD for a nonisotropic scattering scenario.

Fig. 6(a) and (b) depicts the theoretical LCRs and AFDs for different VTDs (low or high) in V2V Ricean fading channels when the Tx and Rx move in the SD, respectively. For further comparison, the measured results taken from [26] are also plotted in Fig. 6(a) and (b). In [26], the measurement campaigns were performed at a carrier frequency of $5.2 \mathrm{GHz}$ on a highway with a low VTD in Germany, and the maximum Doppler frequencies were $f_{T_{\max }}=f_{R_{\max }}=500 \mathrm{~Hz}$. The distance between 


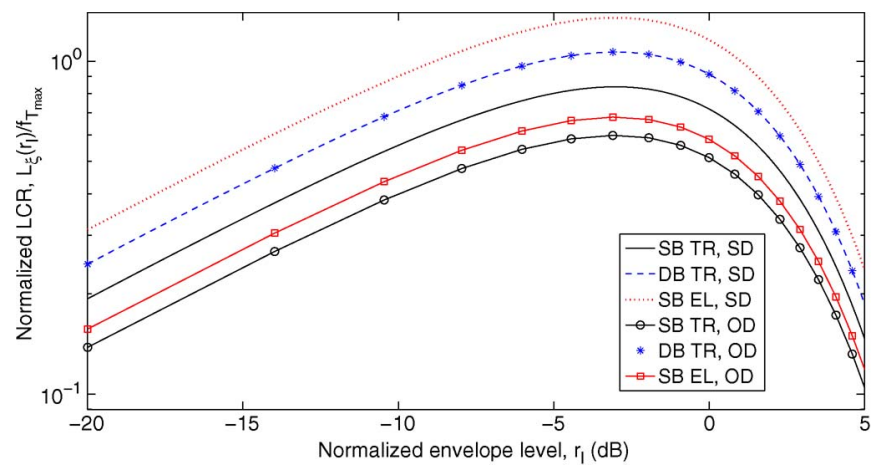

Fig. 4. LCRs of the SB TR model, DB TR model, and SB EL model for different directions of movement of the Tx and $\mathrm{Rx}$ in a V2V nonisotropic scenario $\left(k_{T}^{\mathrm{TR}}=k_{R}^{\mathrm{TR}}=k_{R}^{\mathrm{EL}}=3, \mu_{T}^{\mathrm{TR}}=45^{\circ}\right.$, and $\left.\mu_{R}^{\mathrm{TR}}=\mu_{R}^{\mathrm{EL}}=145^{\circ}\right)$. SD: $\left(\gamma_{T}=\gamma_{T}^{\prime}=\gamma_{R}=\gamma_{R}^{\prime}=0\right)$; OD: $\left(\gamma_{T}=\gamma_{T}^{\prime}=0\right.$ and $\left.\gamma_{R}=\gamma_{R}^{\prime}=\pi\right)$.

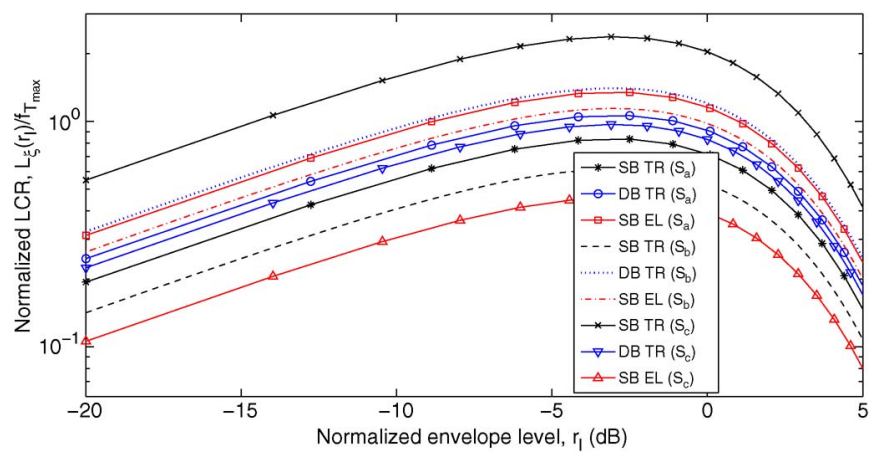

Fig. 5. LCRs of the SB TR model, DB TR model, and SB EL model in $\mathrm{V} 2 \mathrm{~V}$ channels with the Tx and Rx moving in the same direction $\left(\gamma_{T}=\gamma_{T}^{\prime}=\right.$ $\left.\gamma_{R}=\gamma_{R}^{\prime}=0\right)$ for different nonisotropic scenarios. Scenario $a\left(S_{a}\right): k_{T}^{\mathrm{TR}}=$ $k_{R}^{\mathrm{TR}}=k_{R}^{\mathrm{EL}}=3, \mu_{T}^{\mathrm{TR}}=45^{\circ}$, and $\mu_{R}^{\mathrm{TR}}=\mu_{R}^{\mathrm{EL}}=145^{\circ}$. Scenario $b\left(S_{b}\right)$ : $k_{T}^{\mathrm{TR}}=k_{R}^{\mathrm{TR}}=k_{R}^{\mathrm{EL}}=6, \mu_{T}^{\mathrm{TR}}=45^{\circ}$, and $\mu_{R}^{\mathrm{TR}}=\mu_{R}^{\mathrm{EL}}=145^{\circ}$. Scenario $c$ $\left(S_{c}\right): k_{T}^{\mathrm{TR}}=k_{R}^{\mathrm{TR}}=k_{R}^{\mathrm{EL}}=3, \mu_{T}^{\mathrm{TR}}=120^{\circ}, \mu_{R}^{\mathrm{TR}}=45^{\circ}$, and $\mu_{R}^{\mathrm{EL}}=0^{\circ}$.

the Tx and Rx was approximately $D=300 \mathrm{~m}$, and the directions of movement of the Tx and Rx were $\gamma_{T}=\gamma_{R}=0$ (SD). Both Tx and Rx were equipped with one omnidirectional antenna, i.e., SISO case. Based on the measured scenarios in [26], we chose the following environment-related parameters for Fig. 6(a) and (b): $\mu_{T}^{\mathrm{TR}}=33.2^{\circ}, k_{T}^{\mathrm{TR}}=18.2, \mu_{R}^{\mathrm{TR}}=148.6^{\circ}$, $k_{R}^{\mathrm{TR}}=13.3, \mu_{R}^{\mathrm{ER}}=148.6^{\circ}$, and $k_{R}^{\mathrm{ER}}=8.6$. Considering the constraints of the Ricean factor and power-related parameters for a propagation scenario with low VTD as mentioned in Section II, we assume that the Ricean factor is comparatively large and single-bounced rays of the EL model bear more power than SB and double-bounced rays of the TR model. Based on the minimum of the mean squared error between the theoretical LCR/AFD and the measured LCR/AFD reported in [26], we can obtain the following parameters: $K=4.26$, $\eta_{\mathrm{DB}}=0.08, \eta_{\mathrm{SB}_{1}}=0.12, \eta_{\mathrm{SB}_{2}}=0.18$, and $\eta_{\mathrm{SB}_{3}}=0.62$. The excellent agreement between the theoretical results and measured data confirms the utility of the proposed model. The theoretical LCR and AFD for high VTD shown in Fig. 6(a) and (b) were obtained via the assumption that the Ricean factor is comparatively small and double-bounced rays bear more energy than single-bounced rays, i.e., $K=0.56, \eta_{\mathrm{DB}}=0.58$, $\eta_{\mathrm{SB}_{1}}=0.1, \eta_{\mathrm{SB}_{2}}=0.18$, and $\eta_{\mathrm{SB}_{3}}=0.14$. Unfortunately, to the best of the authors' knowledge, no measurement results are

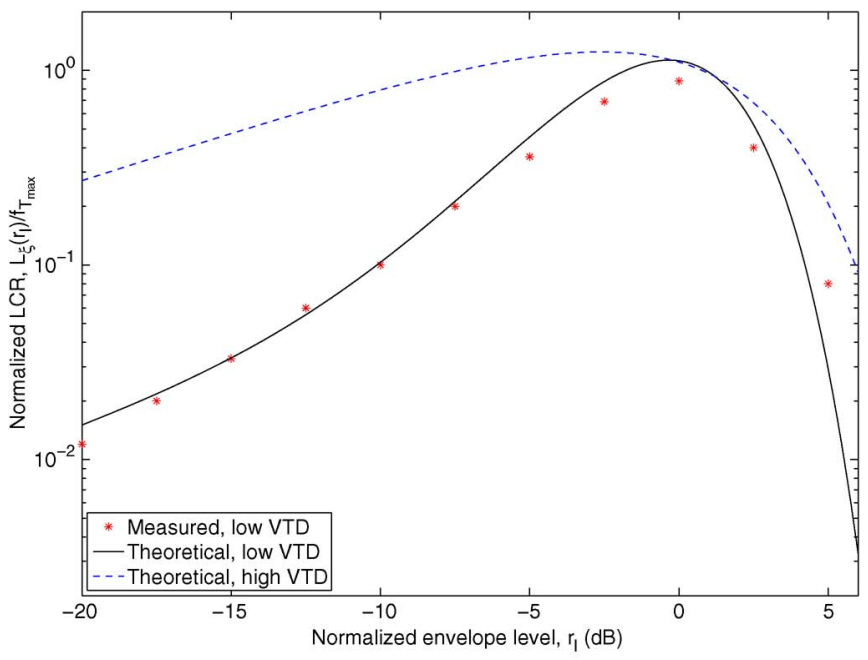

(a)

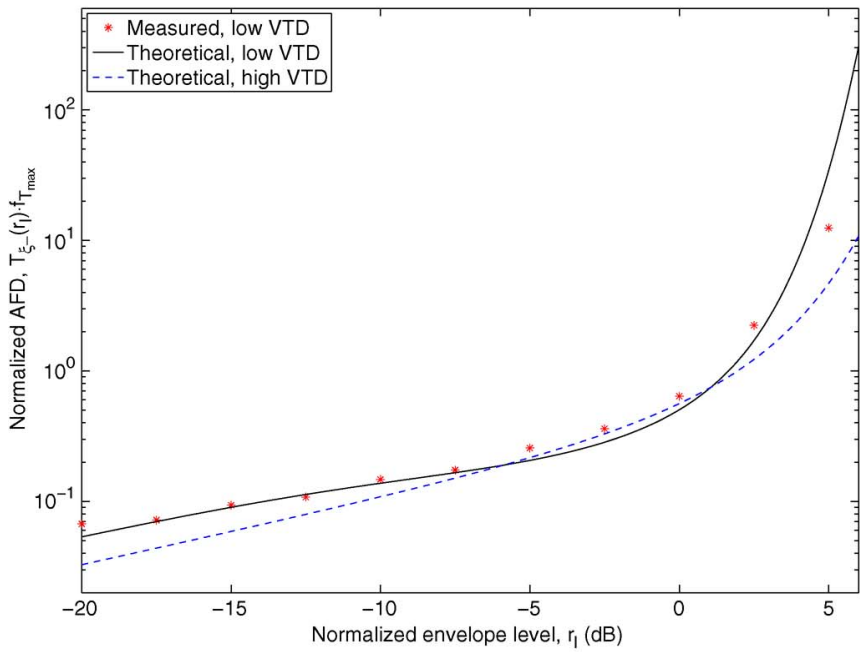

(b)

Fig. 6. (a) LCRs and (b) AFDs of the developed V2V channel model with a low or high VTD when the Tx and Rx move in the same direction.

available for studying the impact of high VTD (e.g., traffic jam) on the LCR and AFD. Comparing the theoretical LCRs and AFDs in Fig. 6(a) and (b), respectively, we observe that the VTD significantly affects the LCR and AFD for V2V channels. However, we note that the LCR and AFD around $r_{l}=0 \mathrm{~dB}$ are nearly independent of the VTD. This attractive property suggests that the LCR can be used to provide a velocity estimate that is robust to the VTD. Moreover, Fig. 6(a) shows that the fades are shallower when the VTD is lower. We see in Fig. 6(b) that the AFD tends to be larger with lower VTD. This is because with a high VTD, the received power comes mainly from the moving cars around the TX and Rx from all directions, whereas with a low VTD, the received power concentrates on several directions, e.g., the directions of LoS components and/or large stationary scatterers on the roadside environment. The above observations coincide with our intuition that the LCR increases with the increase in the VTD while the AFD increases with the decrease in the VTD. This demonstrates the correctness of the method by taking into account the impact of the VTD in our model, i.e., using a TR model to describe the moving scatterers 
around the Tx and Rx and to utilize an EL model to depict the stationary scatterers located on the roadside.

\section{CONCLUSION}

In this paper, a new generic RS-GBSM that can be used to practically study the impact of the VTD on channel statistics has been proposed for a 2-D nonisotropic scattering narrowband SISO V2V Ricean fading channel. Based on the proposed model, the LCR and AFD have been derived and further investigated in more detail. Analysis and simulation results have shown that some important parameters, e.g., the scattering assumption, shape of the scattering region, mean angle, angle spread, and directions of movement of the Tx and Rx, can significantly affect the LCR and AFD of V2V channels. More importantly, we have investigated the impact of VTD on LCR and AFD and found that a lower VTD results in a smaller LCR (i.e., the shallower fades) and correspondingly a larger AFD. In addition, we have found that the LCR and AFD around $r_{l}=0 \mathrm{~dB}$ are roughly independent of the VTD, indicating that the velocity estimate obtained from the LCR is robust against the VTD. Finally, it has been shown that the theoretical results of the LCR and AFD match the measured data, which validates the utility of the analysis and channel model proposed in this paper in the practical design of $\mathrm{V} 2 \mathrm{~V}$ communications in ITSs.

\section{APPENDIX A \\ DERIVATION OF (8B)}

The starting point for the derivation of (8b) is given by the time ACF of the complex channel fading envelope $h^{\mathrm{DB}}(t)$. Substituting (2c) into the expression of $\rho_{h^{\mathrm{DIF}}}(\tau)$, considering the von Mises PDFs for TR scatterers and using equality $\int_{-\pi}^{\pi} e^{a \sin c+b \cos c} d c=2 \pi I_{0}\left(\sqrt{a^{2}+b^{2}}\right)$ [29], we can obtain the closed-form expression of $\rho_{h^{\mathrm{DB}}}(\tau)$ as

$$
\begin{aligned}
& \rho_{h^{\mathrm{DB}}}(\tau)=\frac{\eta_{\mathrm{DB}}}{K+1} \frac{1}{I_{0}\left(k_{T}^{\mathrm{TR}}\right) I_{0}\left(k_{R}^{\mathrm{TR}}\right)} \\
& \quad \times I_{0}\left\{\sqrt{\left(A_{T}^{\mathrm{DB}}\right)^{2}+\left(B_{T}^{\mathrm{DB}}\right)^{2}}\right\} I_{0}\left\{\sqrt{\left(A_{R}^{\mathrm{DB}}\right)^{2}+\left(B_{R}^{\mathrm{DB}}\right)^{2}}\right\}
\end{aligned}
$$

where $A_{T}^{\mathrm{DB}}=k_{T}^{\mathrm{TR}} \cos \mu_{T}^{\mathrm{TR}}+j 2 \pi \tau f_{T_{\max }} \cos \gamma_{T}, B_{T}^{\mathrm{DB}}=k_{T}^{\mathrm{TR}}$ $\sin \mu_{T}^{\mathrm{TR}}+j 2 \pi \tau f_{T_{\max }} \sin \gamma_{T}, A_{R}^{\mathrm{DB}}=k_{R}^{\mathrm{TR}} \cos \mu_{R}^{\mathrm{TR}}+j 2 \pi \tau f_{R_{\max }}$ $\cos \gamma_{R}$, and $B_{R}^{\mathrm{DB}}=k_{R}^{\mathrm{TR}} \sin \mu_{R}^{\mathrm{TR}}+j 2 \pi \tau f_{R_{\max }} \sin \gamma_{R}$. Substituting (13) into the expression of $b_{m}$ with $m=2$ and applying trigonometric transformations, product rule for differentiation, and equalities $d I_{V}(z) / d z=\left[I_{V-1}(z)+I_{V+1}(z)\right] / 2$ and $z I_{V-1}(z)-z I_{V+1}(z)=(V+1) I_{V}(z)$ [29], where $I_{V}(\cdot)$ is the $V$ th-order modified Bessel function of the first kind, we can get the closed-form expression of the parameter $b_{2}^{\mathrm{DB}}$ given in (8b) after extensive manipulations.

\section{APPENDIX B}

\section{DERIVATION OF (10B)}

Note that (10b) includes two formulas regarding parameters $b_{2}^{\mathrm{SB}_{1}}$ and $b_{2}^{\mathrm{SB}_{2}}$. In this appendix, we only derive parameter $b_{2}^{\mathrm{SB}_{1}}$ since the derivation of parameter $b_{2}^{\mathrm{SB}_{2}}$ is exactly the same. Different from the derivation of the parameter $b_{2}^{\mathrm{DB}}$ given in Appendix A, we derive parameter $b_{2}^{\mathrm{SB}_{1}}$ directly from its general expression in (9a) with $m=2$ in (14), shown at the top of the next page. For the above equation, the first term $X_{1}$ can be readily obtained as the following closed-form expression based on equality $\int_{-\pi}^{\pi} e^{a \sin c+b \cos c} d c=2 \pi I_{0}\left(\sqrt{a^{2}+b^{2}}\right)$ [29]:

$$
\begin{aligned}
X_{1}=b_{0}^{\mathrm{SB}_{1}} 4 \pi^{2}\left(\frac{f_{T_{\max }}^{2}}{2}\right. & +f_{R_{\max }}^{2} \cos ^{2} \gamma_{R}+\frac{f_{R_{\max }}^{2} \Delta_{T}^{2} \sin ^{2} \gamma_{R}}{2} \\
& \left.+f_{T_{\max }} f_{R_{\max }} \Delta_{T} \sin \gamma_{T} \sin \gamma_{R}\right)
\end{aligned}
$$

The second term $X_{2}$ is given as follows:

$$
\begin{aligned}
X_{2}= & \frac{b_{0}^{\mathrm{SB}_{1}} 4 \pi f_{T_{\max }} f_{R_{\max }} \cos \gamma_{R}}{I_{0}\left(k_{T}^{\mathrm{TR}}\right)} \int_{-\pi}^{\pi} e^{k_{T}^{\mathrm{TR}} \cos \left(\phi_{T}^{\mathrm{SB}}-_{T}^{\mathrm{TR}}\right)} \\
& \times \frac{1}{2}\left[e^{j\left(\phi_{T}^{\mathrm{SB}_{1}}-\gamma_{T}\right)}+e^{-j\left(\phi_{T}^{\mathrm{SB}}-\gamma_{T}\right)}\right] d \phi_{T}^{\mathrm{SB}_{1}} \\
= & \frac{b_{0}^{\mathrm{SB}} 2 \pi f_{T_{\max }} f_{R_{\max }} \cos \gamma_{R}}{I_{0}\left(k_{T}^{\mathrm{TR}}\right)} \\
& \times\left[e_{-\pi / 2+\mu_{T}^{\mathrm{TR}}}^{j\left(\pi / 2+\mu_{T}^{\mathrm{TR}}-\gamma_{T}\right)} \int_{3 \pi / 2-\mu_{T}^{\mathrm{TR}}}^{3 \pi / 2+\mu_{T}^{\mathrm{TR}}} e_{-\pi / 2-\mu_{T}^{\mathrm{TR}}}^{-j \beta_{T}+k_{T}^{\mathrm{TR}} \sin \beta_{T}} d \beta_{T}\right.
\end{aligned}
$$

where in the third equality we have used equalities $\phi_{T}^{\mathrm{SB}_{1}}=\pi / 2-$ $\beta_{T}+\mu_{T}^{\mathrm{TR}}$ and $\phi_{T}^{\mathrm{SB}_{1}}=-\pi / 2+\theta_{T}+\mu_{T}^{\mathrm{TR}}$ so that $d \phi_{T}^{\mathrm{SB}_{1}}=$ $-d \beta_{T}$ and $d \phi_{T}^{\mathrm{SB}_{1}}=d \theta_{T}$ hold, respectively. To solve the definite integrals in the right-hand side of (16), we need to modify the following equality: $\int_{-\pi}^{\pi} e^{-j V \theta+j z \sin \theta} d \theta=2 \pi J_{V}(z)$ [29], where $J_{V}(\cdot)$ is the $V$ th-order Bessel function of the first kind. Considering the periodic property of the exponential function in the left-hand side of the above equation (here, its period is $2 \pi)$ and $J_{V}(-j z)=(-j)^{V} I_{V}(z)$, we can extend the aforementioned equality into a more general expression as $\int_{\alpha_{l}}^{\alpha_{u}} e^{-j V \theta+z \sin \theta} d \theta=2 \pi(-j)^{V} I_{V}(z)$, where $\left|\alpha_{u}-\alpha_{l}\right|=$ $2 \pi$. It follows that (16) becomes the following closed-form expression:

$$
X_{2}=b_{0}^{\mathrm{SB}} 8 \pi^{2} f_{T_{\max }} f_{R_{\max }} \cos \gamma_{R} \cos \left(\gamma_{T}-\mu_{T}^{\mathrm{TR}}\right) \frac{I_{1}\left(k_{T}^{\mathrm{TR}}\right)}{I_{0}\left(k_{T}^{\mathrm{TR}}\right)} .
$$

Using the same procedure shown above, the closed-form expressions of other terms $X_{3}, X_{4}, X_{5}$, and $X_{6}$ can be obtained similarly. The substitution of $X_{1}, X_{2}, \ldots, X_{6}$ into (14) gives the final result of $b_{2}^{\mathrm{SB}_{1}}$, as shown in (10b). 


$$
\begin{aligned}
& b_{2}^{\mathrm{SB}_{1}}=\frac{b_{0}^{\mathrm{SB}_{1}} 2 \pi}{I_{0}\left(k_{T}^{\mathrm{TR}}\right)} \int_{-\pi}^{\pi} e^{k_{T}^{\mathrm{TR}} \cos \left(\phi_{T}^{\mathrm{SB}_{1}}-\mu_{T}^{\mathrm{TR}}\right)} \\
& \times\left(\frac{f_{T_{\max }}^{2}}{2}+\frac{f_{R_{\max }}^{2} \Delta_{T}^{2} \sin ^{2} \gamma_{R}}{2}+f_{R_{\max }}^{2} \cos ^{2} \gamma_{R}+f_{T_{\max }} f_{R_{\max }} \Delta_{T} \sin \gamma_{T} \times \sin \gamma_{R}\right) d \phi_{T}^{\mathrm{SB}} \\
& -\left[\frac{b_{0}^{\mathrm{SB}_{1}} 2 \pi}{I_{0}\left(k_{T}^{\mathrm{TR}}\right)} \int_{-\pi}^{\pi} e^{k_{T}^{\mathrm{TR}} \cos \left(\phi_{T}^{\mathrm{SB}_{1}}-\mu_{T}^{\mathrm{TR}}\right)} 2 f_{T_{\max }} f_{R_{\max }} \cos \gamma_{R} \cos \left(\phi_{T}^{\mathrm{SB}_{1}}-\gamma_{T}\right) d \phi_{T}^{\mathrm{SB}_{1}}+\frac{b_{0}^{\mathrm{SB}_{1}} 2 \pi}{I_{0}\left(k_{T}^{\mathrm{TR}}\right)}\right. \\
& \left.\times \int_{-\pi}^{\pi} e^{k_{T}^{\mathrm{TR}} \cos \left(\phi_{T}^{2 S B_{1}}-\mu_{T}^{\mathrm{TR}}\right)} f_{R_{\max }}^{2} \Delta_{T} \sin \left(2 \gamma_{R}\right) \sin \phi_{T}^{\mathrm{SB}_{1}} d \phi_{T}^{\mathrm{SB}_{1}}\right] \\
& +\left[\frac{b_{0}^{\mathrm{SB}_{1}} 2 \pi}{I_{0}\left(k_{T}^{\mathrm{TR}}\right)} \int_{-\pi}^{\pi} e^{k_{T}^{\mathrm{TR}} \cos \left(\phi_{T}^{\mathrm{SB}_{1}}-\mu_{T}^{\mathrm{TR}}\right)} f_{T_{\max }}^{2} \frac{\cos \left(2\left(\phi_{T}^{\mathrm{SB}_{1}}-\gamma_{T}\right)\right)}{2} d \phi_{T}^{\mathrm{SB}_{1}}\right. \\
& -\frac{b_{0}^{\mathrm{SB}_{1}} 2 \pi}{I_{0}\left(k_{T}^{\mathrm{TR}}\right)} \int_{-\pi}^{\pi} e^{k_{T}^{\mathrm{TR}} \cos \left(\phi_{T}^{\mathrm{SB}_{1}}-\mu_{T}^{\mathrm{TR}}\right)} \frac{f_{R_{\max }}^{2} \Delta_{T}^{2} \sin ^{2} \gamma_{R} \cos \left(2 \phi_{T}^{\mathrm{SB}_{1}}\right)}{2} d \phi_{T}^{\mathrm{SB}_{1}} \\
& \left.+\frac{b_{0}^{\mathrm{SB}_{1}} 2 \pi}{I_{0}\left(k_{T}^{\mathrm{TR}}\right)} \int_{-\pi}^{\pi} e^{k_{T}^{\mathrm{TR}}} \cos \left(\phi_{T}^{\mathrm{SB}_{1}}-\mu_{T}^{\mathrm{TR}}\right) f_{T_{\max }} f_{R_{\max }} \Delta_{T} \sin \gamma_{R} \sin \left(\gamma_{T}-2 \phi_{T}^{\mathrm{SB}_{1}}\right) d \phi_{T}^{\mathrm{SB}_{1}}\right] \\
& =X_{1}-\left[X_{2}+X_{3}\right]+\left[X_{4}-X_{5}+X_{6}\right] \text {. }
\end{aligned}
$$

\section{REFERENCES}

[1] S. T. Cheng, G. J. Horng, and C. L. Chou, "Using cellular automata to form car society in vehicular ad-hoc networks," IEEE Trans. Intell. Transp. Syst., vol. 12, no. 4, pp. 1374-1384, Dec. 2011.

[2] L. Yang and F. Wang, "Driving into intelligent spaces with pervasive computing and communications," IEEE Intell. Syst., vol. 22, no. 1, pp. $12-$ 15, Jan./Feb. 2007.

[3] X. Cheng, C.-X. Wang, H. Wang, X. Gao, X.-H. You, D. Yuan, B. Ai, Q. Huo, L. Song, and B. Jiao, "Cooperative MIMO channel modeling and multi-link spatial correlation properties," IEEE J. Sel. Areas Commun., vol. 30, no. 2, pp. 388-396, Feb. 2012.

[4] Standard for Wireless Local Area Networks Providing Wireless Communications While in Vehicular Environment, IEEE P802.11p/D2.01, Mar. 2007.

[5] D. Duan, L. Yang, and J. C. Principe, "Cooperative diversity of spectrum sensing for cognitive radio systems," IEEE Trans. Signal Process., vol. 58, no. 6, pp. 3218-3227, Jun. 2010.

[6] W. Cho and L. Yang, "Optimum resource allocation for relay networks with differential modulation," IEEE Trans. Commun., vol. 56, no. 4, pp. 531-534, Apr. 2008.

[7] C.-X. Wang, X. Hong, X. Ge, X. Cheng, G. Zhang, and J. S. Thompson, "Cooperative MIMO channel models: A survey," IEEE Commun. Mag., vol. 48, no. 2, pp. 80-87, Feb. 2010.

[8] R. Zhang, X. Cheng, Q. Yao, C.-X. Wang, Y. Yang, and B. Jiao, "Interference graph based resource sharing schemes for vehicular networks," IEEE Trans. Veh. Technol., vol. 62, no. 8, pp. 4028-4039, Oct. 2013.

[9] C.-X. Wang, X. Cheng, and D. I. Laurenson, "Vehicle-to-vehicle channel modeling and measurements: Recent advances and future challenges," IEEE Commun. Mag., vol. 47, no. 11, pp. 96-103, Nov. 2009.

[10] I. Sen and D. W. Matolak, "Vehicle-vehicle channel models for the 5-GHz band," IEEE Trans. Intell. Transp. Syst., vol. 9, no. 2, pp. 235245, Jun. 2008.

[11] A. S. Akki and F. Haber, "A statistical model for mobile-to-mobile land communication channel," IEEE Trans. Veh. Technol., vol. VT-35, no. 1, pp. 2-7, Feb. 1986.

[12] A. G. Zaji and G. L. Stüber, "Space-time correlated mobile-to-mobile channels: Modelling and simulation," IEEE Trans. Veh. Technol., vol. 57, no. 2, pp. 715-726, Mar. 2008.
[13] X. Cheng, Q. Yao, C.-X. Wang, B. Ai, G. L. Stuber, D. Yuan, and B. Jiao, "An improved parameter computation method for a MIMO V2V Rayleigh fading channel simulator under non-isotropic scattering environments," IEEE Commun. Lett., vol. 17, no. 2, pp. 265-268, Feb. 2013.

[14] A. G. Zaji and G. L. Stüber, "Three-dimensional modeling, simulation, and capacity analysis of space-time correlated mobile-to-mobile channels," IEEE Trans. Veh. Technol., vol. 57, no. 4, pp. 2042-2054, Jul. 2008.

[15] A. G. Zaji and G. L. Stüber, "Three-dimensional modeling and simulation of wideband MIMO mobile-to-mobile channels," IEEE Trans. Wireless Commun., vol. 8, no. 3, pp. 1260-1275, Mar. 2009.

[16] X. Cheng, C.-X. Wang, D. I. Laurenson, S. Salous, and A. V. Vasilakos, "An adaptive geometry-based stochastic model for non-isotropic MIMO mobile-to-mobile channels," IEEE Trans. Wireless Commun., vol. 8, no. 9, pp. 4824-4835, Sep. 2009.

[17] X. Cheng, Q. Yao, M. Wen, C.-X. Wang, L. Song, and B. Jiao, "Wideband channel modeling and ICI cancellation for vehicle-to-vehicle communication systems," IEEE J. Sel. Areas Commun., vol. 31, no. 8, Aug. 2013.

[18] A. S. Akki, "Statistical properties of mobile-to-mobile land communication channels," IEEE Trans. Veh. Technol., vol. 43, no. 4, pp. 826-831, Nov. 1994.

[19] A. G. Zaji, G. L. Stüber, T. G. Pratt, and S. Nguyen, "Envelope level crossing rate and average fade duration in mobile-to-mobile fading channels," in Proc. IEEE ICC, Beijing, China, May 2008, pp. 4446-4450.

[20] A. G. Zaji, G. L. Stüber, T. G. Pratt, and S. Nguyen, "Wideband MIMO mobile-to-mobile channels: Geometry-based statistical modeling with experimental verification," IEEE Trans. Veh. Technol., vol. 58, no. 2, pp. 517-534, Feb. 2009.

[21] X. Cheng, C.-X. Wang, D. I. Laurenson, and A. V. Vasilakos, "Second order statistics of non-isotropic mobile-to-mobile Ricean fading channels," in Proc. IEEE ICC, Dresden, Germany, Jun. 2009, pp. 1-5.

[22] G. L. Stüber, Principles of Mobile Communication, 2nd ed. Boston, MA, USA: Kluwer, 2001

[23] M. Pätzold, U. Killat, and F. Laue, "An extended Suzuki model for land mobile satellite channels and its statistical properties," IEEE Trans. Veh. Technol., vol. 47, no. 2, pp. 617-630, May 1998.

[24] S. O. Rice, "Distribution of the duration of fades in radio transmission: Gaussian noise model," Bell Syst. Technol. J., vol. 37, no. 3, pp. 581-635, May 1958 
[25] S. Wang, A. Abdi, J. Salo, H. M. EL-Sallabi, J. W. Wallace, P. Vainikainen, and M. A. Jensen, "Time-varying MIMO channels: Parametric statistical modeling and experimental results," IEEE Trans. Veh. Technol., vol. 56, no. 4, pp. 1949-1963, Jul. 2007.

[26] J. Maurer, T. Fügen, and W. Wisebeck, "Narrow-band measurement and analysis of the inter-vehicle transmission channel at $5.2 \mathrm{GHz}$," in Proc. IEEE VTC-Spring, Birmingham, AL, USA, May 2002, pp. 1274-1278.

[27] A. Abdi, J. A. Barger, and M. Kaveh, "A parametric model for the distribution of the angle of arrival and the associated correlation function and power spectrum at the mobile station," IEEE Trans. Veh. Technol., vol. 51, no. 3, pp. 425-434, May 2002.

[28] N. Youssef, C.-X. Wang, and M. Pätzold, "A study on the second order statistics of Nakagami-Hoyt mobile fading channels," IEEE Trans. Veh. Technol., vol. 54, no. 4, pp. 1259-1265, Jul. 2005.

[29] I. S. Gradshteyn and I. M. Ryzhik, Table of Integrals, Series, and Products 6th. Boston, MA, USA: Academic, 2000.

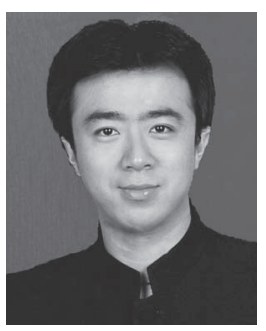

Xiang Cheng (S'05-M'10-SM'13) received the Ph.D. degree from Heriot-Watt University, Edinburgh, U.K., and The University of Edinburgh, Edinburgh, in 2009.

He has been with Peking University, Beijing, China, since 2010, first as a Lecturer and then as an Associate Professor since 2012. He has published more than 60 research papers in journals and conference proceedings. His research interests include mobile propagation channel modeling and simulation, next-generation mobile cellular systems, intelligent transportation systems (ITS), and hardware prototype development.

Dr. Cheng served as a Symposium Cochair and a Member of Technical Program Committee for several international conferences. He was a recipient of the Best Paper Award from the IEEE International Conference on ITS Telecommunications 2012, the "2009 Chinese National Award for Outstanding Overseas $\mathrm{Ph} . D$. Students" for his academic excellence and outstanding performance, and the Postgraduate Research Thesis Prize from The University of Edinburgh.

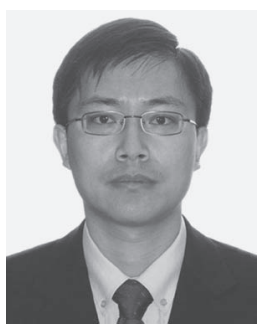

Cheng-Xiang Wang (S'01-M'05-SM'08) received the B.Sc. and M.Eng. degrees in communication and information systems from Shandong University, Jinan, China, in 1997 and 2000, respectively, and the Ph.D. degree in wireless communications from Aalborg University, Aalborg, Denmark, in 2004.

Since 2005, he has been with Heriot-Watt University, Edinburgh, U.K., first as a Lecturer, then as a Reader in 2009, and was promoted to Professor in 2011. He is also an Honorary Fellow of The University of Edinburgh, Edinburgh, and a Chair/Guest Professor at Shandong University; Huazhong University of Science and Technology, Wuhan, China; and Southeast University, Nanjing, China. He was a Research Fellow at the University of Agder, Grimstad, Norway, from 2001 to 2005; a Visiting Researcher at Siemens AG-Mobile Phones, Munich, Germany, in 2004; and a Research Assistant at the Technical University of HamburgHarburg, Hamburg, Germany, from 2000 to 2001. He has edited one book and published one book chapter and more than 180 papers in refereed journals and conference proceedings. His research interests include wireless channel modeling and simulation, green communications, cognitive radio networks, vehicular communication networks, large multiple-input multiple-output (MIMO), cooperative MIMO, and $\mathrm{B} 4 \mathrm{G}$ wireless communications.

Prof. Wang is a Fellow of The Institution of Engineering and Technology, a Fellow of the Higher Education Academy, and a member of the Engineering and Physical Sciences Research Council Peer Review College. He served or is currently serving as a Technical Program Committee (TPC) member, TPC Chair, and General Chair for more than 70 international conferences. He served or is currently serving as an editor for eight international journals, including the IEEE TRANSACTIONS ON VeHICULAR TECHNOLOGY (since 2011) and the IEEE TRANSACTIONS ON Wireless COMMUNiCATions (2007-2009). $\mathrm{He}$ was the leading Guest Editor of the IEEE Journal ON SELECTED AREas in Communications, Special Issue on Vehicular Communications and Networks. He was a recipient of the Best Paper Awards from the IEEE GLOBECOM 2010, the IEEE International Conference on Communication Technology 2011, and the International Conference on Intelligent Transportation Systems Telecommunications 2012.

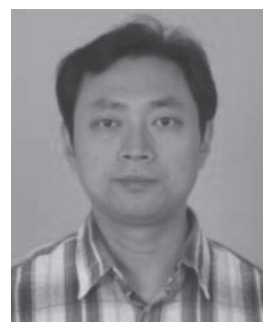

Bo Ai (M'00-SM'09) received the Master's and $\mathrm{Ph} . \mathrm{D}$. degrees from Xidian University, Xi'an, China, in 2002 and 2004, respectively. He graduated in 2007 with great honors of Excellent Postdoctoral Research Fellow from Tsinghua University, Beijing, China.

$\mathrm{He}$ is with Beijing Jiaotong University, Beijing, as a Professor and Advisor of Ph.D. candidates. He is a Deputy Director of the State Key Laboratory of Rail Traffic Control and Safety. He has authored/ coauthored six books, 26 invention patents, and 130 scientific research papers in his research area. His interests are the research and applications of orthogonal frequency-division multiplexing techniques, high-power-amplifier linearization techniques, radio propagation and channel modeling, and Global System for Mobile Communications railway systems.

Dr. Ai is a Senior Member of the Chinese Institute of Electronics.

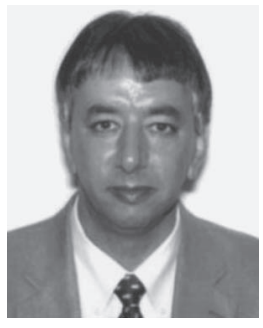

Hadi Aggoune (S'84-M'90-SM'93) received the M.S. and Ph.D. degrees in electrical engineering from the University of Washington (UW), Seattle, WA, USA, in 1984 and 1988, respectively.

He has taught graduate and undergraduate courses in electrical engineering at several universities in the United States and abroad. He served at many academic ranks, including Endowed Chair Professor and Vice President and Provost. Currently, he is a Professor and the Director of the Sensor Networks and Cellular Systems Research Center, University of Tabuk, Tabuk, Saudi Arabia. He authored many papers in IEEE and other journals and conferences. He is listed as Inventor in a major patent assigned to Boeing Company. His research work is referred to in many patents, including patents assigned to ABB, Switzerland, and the Electric Power Research Institute, USA. His research interests include the modeling and simulation of large-scale networks, sensors and sensor networks, scientific visualization, and control and energy systems.

Dr. Aggoune is a Professional Engineer registered in the State of Washington. He is serving on many technical committees. He was the recipient of the Boeing Supplier Excellence Award and the IEEE Professor of the Year Award, UW branch. 